\title{
Gravitational Wave in the Theory with the Universal Charge
}

\author{
D.L. Khokhlov*
}

Sumy State University, R.-Korsakov St. 2 Sumy 40007 Ukraine

\begin{abstract}
Gravitational interaction is considered with use of the universal charge. Gravitational wave is defined as a tenzor electromagnetic like wave of the Planck mass. Within the Planck time it decays into four hypothetical Planck neutrinos. The quadrupole gravitational emission in the theory with the universal charge is the same as in general relativity. Gravitational radiation as Planck neutrinos does not generate quadrupole oscillations when passing through the detector that may explain non-detection of gravitational waves in the LIGO and other projects.
\end{abstract}

Keywords: Gravitational wave, universal charge, decay of proton at the Planck scale.

\section{INTRODUCTION}

In general relativity [1] gravitational wave in empty space is defined by the absence of the matter energymomentum tensor, $T_{i k}=0$, then the Einstein equations reduce to the Ricci tensor equal to zero, $R_{i k}=0$. The energy-momentum conservation law is given by

$$
\frac{\partial}{\partial x^{k}}(-g)\left(T^{i k}+t^{i k}\right)=0
$$

where $t^{i k}$ is the pseudo-tensor for the energy-momentum of gravitational wave. The flux of $t^{i k}$ yields the loss of energy due to the quadrupole gravitational emission [2].

$$
-\frac{d E}{d t}=\frac{G}{45 c^{5}} \frac{d}{d t^{3}}\left(D_{i k}^{2}\right)
$$

where $G$ is the Newton constant, $c$ is the speed of light, $D_{i k}$ is the tensor for quadrupole mass moment. Observations [3] showed that the time decrease of the revolution period of the binary radiopulsar PSR1913+16 is in accordance with the loss of energy for the gravitational emission eq. (2). This is considered as an indirect evidence for the existence of the gravitational waves. However, the LIGO project and its international counterparts have not detected gravitational waves [4].

From the beginning, the concept of gravitational wave faces fundamental difficulties, with the main is that the energy-momentum of gravitational wave is defined by the pseudo-tensor [5]. The ongoing discussion of the problems may be found in $[6,7]$ and references therein.

Despite the problems with interpretation of gravitational wave the modern textbooks [1] consider gravitational wave within the framework of linearized theory in the

*Address correspondence to this author at the Sumy State University, R.Korsakov St. 2 Sumy 40007 Ukraine; Tel: 380542 332558;

Fax: 380542 771063; E-mail: dlkhokhl@ rambler.ru flat background space. Equations for the gravitational wave in vacuum are given by

$$
\square h_{i}^{k}=0 .
$$

The gravitational wave propagates with the speed of light. The tenzor field $h_{i}^{k}$ is a weak perturbation of a Galilean metric

$$
g_{i k}=\eta_{i k}+h_{i k}
$$

with the Lorentz gauge

$$
\frac{\partial \psi_{i}^{k}}{\partial x^{k}}=0, \quad \psi_{i}^{k}=h_{i}^{k}-\frac{1}{2} \delta_{i}^{k} h .
$$

The effective energy-momentum tenzor of the gravitational wave is given by

$$
T_{\mu v}=\frac{c^{4}}{32 \pi G}\left\langle h_{j k, \mu}^{T T} h_{j k, v}^{T T}\right\rangle
$$

where \langle\rangle denotes average over several wavelengths, $h_{j k}^{T T}$ is the transverse traceless part of $h_{\mu v}$ which in TT gauge coincides with $h_{j k}$. Quantization of $h_{j k}$ yields massless field of spin 2. In addition, there were developed extended theories of gravity like scalar-tenzor gravity, bimetric theory, high order theories, Brans-Dicke theory and string theory, e.g. [8] and references therein. In extended theories of gravity gravitational waves could have a third massive polarization which propagates with a speed slower than the speed of light, see discussion in [8] and references therein.

Within the framework of quantum field theory [9] the intensity of electromagnetic, weak and strong interactions is defined by the universal charge, with the different factors for each interaction. This implies a unification theory of all the interactions. To include gravitational interaction in the unification theory one should develop the theory of gravitation with use of the universal charge. The Newton constant may be cast in the form 


$$
G=\frac{g^{2}}{m_{P l}^{2}}
$$

where $g=(\hbar c)^{1 / 2}$ is the universal charge, $m_{P l}=(\hbar c / G)^{1 / 2}$ is the Planck mass, $\hbar$ is the Planck constant. The electromagnetic charge $e$ (charge of electron) and the charge $g$ are related via the fine structure constant as $e^{2}=\alpha g^{2}$. It is worth noting that the effective electromagnetic charge is a growing function of the transferred momentum $q$. Within the framework of quantum field theory [9] the representation of the Newton constant eq. (7) may be interpreted such that the gravitational interaction is governed by the universal charge at the Planck scale. The interaction occurs via the intermediate field of the Planck mass. We shall consider the theory of gravitation with the universal charge $g$. To this end, the concept of the universal charge $g$ was under discussion, e.g. $[10,11]$ and references therein.

Assume that all fermions carry the universal charge $g$. Consider a toy model of proton consisting of positron and four hypothetical partons

$$
p=e^{+} g g g g
$$

where $g$ denotes the parton of the charge $g$ and spin $1 / 2$. Suppose that the total spin of four partons is equal zero and the total momentum of four partons in the frame of a proton is equal zero. It is worth noting that in the theory of strong interaction [9] proton is considered as a combination of three quarks. We shall not touch the question of the interplay of quarks and partons of charge $g$.

Consider interaction of four partons via the intermediate field of the Planck mass of spin 2. We shall consider the interaction of the partons separated by the Compton radius of proton, $r_{c}=\hbar / m_{p} c$. Since the total momentum of four partons in the frame of a proton is zero one can think of the intermediate field as a combination of two vector fields with the opposite momenta, with null total momentum of the intermediate field. That is one can interpret the intermediate field as a standing wave, $\vec{A}_{l} \vec{A}^{m}\left(e^{i k r}+e^{-i k r}\right) / \sqrt{2}$. Then, the propagator of the intermediate field reduces to

$$
\frac{g^{\lambda \sigma}-q^{\lambda} q^{\sigma} / m_{P l}^{2}}{q^{2}-m_{P l}^{2}} \rightarrow \frac{g^{\lambda \sigma}}{m_{P l}^{2}} .
$$

In view of eq. (9) the Lagrangian of interaction of four partons at the Compton radius of proton governed by the universal charge at the Planck scale may be cast in the form

$$
L=\frac{g^{2} m_{p}^{3}}{m_{P l}^{2}} \Psi_{2}^{\dagger} \Psi_{1} \Psi_{4}^{\dagger} \Psi_{3}=G m_{p}^{3} \Psi_{2}^{\dagger} \Psi_{1} \Psi_{4}^{\dagger} \Psi_{3}
$$

where $\Psi$ denotes the wave function of parton, $\hbar=c=1$. The Lagrangian eq. (10) may describe self-gravity of proton. In this case the intermediate field is not free but accompanying the proton. The interaction may be interpreted in terms of the effective charge $g m_{p} / m_{P l}$. In the classical theory one can think of the Newtonian gravity as an interaction of the effective charges $g m_{p} / m_{P l}$.

Assume that the proton may decay into some hypothetical field (call it Planck neutrino) via the free intermediate field of the Planck mass

$$
e^{+} g g g g \rightarrow e^{+}+P l \rightarrow e^{+}+4 v_{P l}
$$

where $P l$ is the intermediate field of the Planck mass, $v_{P l}$ is the Planck neutrino. The Lagrangian eq. (10) may describe the decay of partons into Planck neutrinos provided that the incoming wave functions denote partons, and the outgoing wave functions denote Planck neutrinos. We shall consider the theory at low energies, $q^{2}<m_{P l}^{2}$, thus handling the Planck field as a virtual one. We shall think of the Planck neutrino as a massless particle of spin $1 / 2$ propagating with the speed of light. As known [9] the standard model regards three sorts of neutrinos being partners of three lepton such as electron, muon, tauon respectively thus forming three generations of leptons. Each sort of neutrino carries the quantum number labeling lepton generation which defines the corresponding current. It is natural to believe that the Planck neutrino carries the quantum number which distinguishes the Planck neutrino from the other three neutrinos, and this number defines the parton to Planck neutrino current.

In general relativity gravitational wave carries gravitational potential. In the theory with the universal charge one can define the tenzor electromagnetic like potential

$$
\phi_{i k}=g k \frac{m}{m_{P l}} \mathrm{e}_{i k}
$$

where $k$ is the wave vector, $\mathrm{e}_{i k}$ is the unity polarization tenzor. The strength of the field is given by

$$
\phi k=g k^{2} \frac{m}{m_{P l}} .
$$

The energy of the field is given by

$$
E=\int \frac{(\phi k)^{2}}{32 \pi} d V=\frac{g^{2} k}{32 \pi} \frac{m^{2}}{m_{P l}^{2}} .
$$

Assume that the gravitational wave carries the tenzor electromagnetic like potential eq. (12), with the potential transverse to the wave vector. Write down equations for the gravitational wave in vacuum in the form

$$
\square \phi_{i k}=0
$$

with the Lorentz gauge

$$
\frac{\partial \phi_{i k}}{\partial x_{k}}=0
$$

One can define the energy-momentum tenzor of the gravitational wave as 


$$
T_{\mu v}=\frac{1}{32 \pi} \phi_{j k, \mu}^{T T} \phi_{j k, v}^{T T}
$$

where $=\phi_{j k}^{T T}$ is the transverse traceless part of $\phi_{\mu v}$ which in TT gauge coincides with $\phi_{j k}$. Thus, we come to the concept of gravitational wave as a tenzor electromagnetic like wave.

Acceleration of the system of the charges $g$ may generate the quadrupole emission at the Planck scale. When considering the quadrupole moment of the effective charge $\mathrm{gm} / \mathrm{m}_{P l}$ one can obtain the formula for quadrupole emission eq. (2) where $D_{i j}$ is the tensor of quadrupole moment of the effective charge $\mathrm{gm} / \mathrm{m}_{P l}$. It is worth noting that for the system of the charges $g$ the dipole emission is absent as the charge to mass ratio is the same for all the charges.

One can identify the above described gravitational wave with the intermediate field of the Planck mass of spin 2 . We consider the linearized theory valid at low energies, $q^{2} \ll m_{P l}^{2}$, thus handling the virtual intermediate field of the Planck mass. Owing to the foregoing reasoning one can use the quadrupole mechanism of generation of the electromagnetic like wave at the Planck scale. Within the Planck time the gravitational wave decays into four Planck neutrinos of spin 1/2. We come to the concept of gravitational radiation as Planck neutrinos. In this approach gravitational emission is a process of transformation of the massive matter into the massless matter.

In the approach proposed the gravitational emission is defined as the quadrupole emission of the charge $g$ at the Planck scale which is the same as the quadrupole gravitational emission in general relativity thus it is in accord with the observations of the binary radiopulsar PSR1913+16 [3]. Gravitational radiation as Planck neutrinos does not generate quadrupole oscillations when passing through the detector. Non-detection of gravitational waves in the LIGO and other projects [4] may be considered as an indirect evidence for the approach proposed. It is reasonable to believe that Planck neutrino does not take part in electromagnetic and weak interactions and does in strong and gravitational interactions. Then, Planck neutrino may be considered as a candidate for the dark matter in the universe.

\section{REFERENCES}

[1] Misner CW, Thorne KS, Wheeler JA. Gravitation. Freeman: San Francisco 1973.

[2] Landau LD, Lifshitz EM. The classical theory of fields, 7th ed Moscow in Russian: Nauka 1988;

[3] Taylor JH, Weisberg JM. Further experimental tests of relativistic gravity using the binary pulsar PSR 1913+16. Astroph J 1989; 345 : 434-50.

[4] Sintes AM. Report on an all-sky LIGO search for periodic gravitational waves in the S4 data. J Phys Conf Ser 2008; 122: 012033.

[5] Pauli W. Theory of Relativity. New York: Pergamon 1958.

[6] Loinger A. On Black Holes and Gravitational Waves. Pavia: La Goliardica Pavese 2002.

[7] Crothers SJ. Certain conseptual anomalies in Einstein's theory of relativity. Prog Phys 2008; 1: 52-7.

[8] Corda C. Interferometric detection of gravitational waves: the definitive test for general relativity. Int J Mod Phys D 2009; 18 2275-82.

[9] Itzykson C, Zuber J-B. Quantum Field Theory. New York: McGraw-Hill 1983.

[10] Motz L. Gauge invariance and the quantization of mass (of gravitational charge). Nuovo Cimento 1972; 12B: 239-255.

[11] Sivaram C. Fundamental interactions in the early universe. Int $\mathbf{J}$ Theor Phys 1994; 33: 2407-13.

Received: May 20, 2011

Revised: June 08, 2011

Accepted: June 10, 2011

(C) D.L. Khokhlov; Licensee Bentham Open.

This is an open access article licensed under the terms of the Creative Commons Attribution Non-Commercial License (http://creativecommons.org/licenses/by-nc/3.0/) which permits unrestricted, non-commercial use, distribution and reproduction in any medium, provided the work is properly cited. 